\title{
Univalency and Convexity Conditions for a General Integral Operator
}

\author{
Serap Bulut ${ }^{1}$ and Daniel Breaz ${ }^{2}$ \\ ${ }^{1}$ Civil Aviation College, Kocaeli University, Arslanbey Campus, İmit, 41285 Kocaeli, Turkey \\ ${ }^{2}$ Department of Mathematics, "1 Decembrie 1918” University of Alba Iulia, Nicolae Iorga Street, No. 11-13, 510000 Alba Iulia, Romania
}

Correspondence should be addressed to Serap Bulut; bulutserap@yahoo.com

Received 24 October 2013; Accepted 26 December 2013; Published 20 February 2014

Academic Editors: F. Uhlig, H. You, and C.-G. Zhu

Copyright (c) 2014 S. Bulut and D. Breaz. This is an open access article distributed under the Creative Commons Attribution License, which permits unrestricted use, distribution, and reproduction in any medium, provided the original work is properly cited.

For analytic functions $f$ and $g$ in the open unit disc $\mathbb{U}$, a new general integral operator is introduced. The main objective of this paper is to obtain univalence condition and order of convexity for this general integral operator.

\section{Introduction and Preliminaries}

Let $\mathscr{A}$ be the class of all functions of the form

$$
f(z)=z+\sum_{k=2}^{\infty} a_{k} z^{k}
$$

which are analytic in the open unit disk

$$
\mathbb{U}=\{z \in \mathbb{C}:|z|<1\} .
$$

Also let $\mathcal{S}$ denote the subclass of $\mathscr{A}$ consisting of functions $f$ which are univalent in $\mathbb{U}$.

A function $f \in \mathscr{A}$ is said to be starlike of order $\alpha(0 \leq$ $\alpha<1$ ) if it satisfies the inequality

$$
\operatorname{Re}\left\{\frac{z f^{\prime}(z)}{f(z)}\right\}>\alpha,
$$

for all $z \in \mathbb{U}$. We say that $f$ is in the class $\mathcal{S}^{*}(\alpha)$ for such functions.

A function $f \in \mathscr{A}$ is said to be convex of order $\alpha(0 \leq \alpha<$ 1) if it satisfies the inequality

$$
\operatorname{Re}\left\{1+\frac{z f^{\prime \prime}(z)}{f^{\prime}(z)}\right\}>\alpha,
$$

for all $z \in \mathbb{U}$. We say that $f$ is in the class $\mathscr{K}(\alpha)$ for such functions.

We note that $f \in \mathscr{K}(\alpha)$ if and only if $z f^{\prime} \in \mathcal{S}^{*}(\alpha)$.
A function $f \in \mathscr{A}$ belongs to the class $\mathscr{R}(\alpha)(0 \leq \alpha<1)$ if it satisfies the inequality

$$
\operatorname{Re}\left\{f^{\prime}(z)\right\}>\alpha,
$$

for all $z \in \mathbb{U}$.

The family $\mathscr{B}(\mu, \alpha)(\mu \geq 0,0 \leq \alpha<1)$ which contains the functions $f$ that satisfy the condition

$$
\left|f^{\prime}(z)\left(\frac{z}{f(z)}\right)^{\mu}-1\right|<1-\alpha \quad(z \in \mathbb{U})
$$

was studied by Frasin and Jahangiri [1].

Remark 1. This family is a comprehensive class of analytic functions that contains other new classes of analytic univalent functions as well as some very well-known ones. For example,

(i) for $\mu=1$, we have the class

$$
\mathscr{B}(1, \alpha)=\mathcal{S}^{*}(\alpha),
$$

(ii) for $\mu=0$, we have the class

$$
\mathscr{B}(0, \alpha)=\mathscr{R}(\alpha),
$$

(iii) for $\mu=2$, the class

$$
\mathscr{B}(\alpha)=\left\{f \in \mathscr{A}:\left|\frac{z^{2} f^{\prime}(z)}{f^{2}(z)}-1\right|<1-\alpha ; 0 \leq \alpha<1 ; z \in \mathbb{U}\right\}
$$

introduced by Frasin and Darus [2]. 
In this paper, we introduce a new general integral operator defined by

$$
I_{n, \alpha}(f, g)(z)=\int_{0}^{z} \prod_{i=1}^{n}\left(f_{i}^{\prime}(t) e^{g_{i}(t)}\right)^{\alpha_{i}} d t,
$$

where $z \in \mathbb{U}, f_{i}, g_{i} \in \mathscr{A}$, and $\alpha_{i} \in \mathbb{C}$ for all $i=1, \ldots, n$.

Remark 2. For $n=1, f_{1}=f, g_{1}=g$, and $\alpha_{1}=\alpha$, we have the integral operator

$$
I_{1}(f, g)(z)=\int_{0}^{z}\left(f^{\prime}(t) e^{g(t)}\right)^{\alpha} d t
$$

introduced by Ularu and Breaz [3].

The following results will be required in our investigation.

General Schwarz Lemma (see [4]). Let the function $f$ be regular in the disk $\mathbb{U}_{R}=\{z \in \mathbb{C}:|z|<R\}$, with $|f(z)|<M$ for fixed $M$. If $f$ has one zero with multiplicity order bigger than $m$ for $z=0$, then

$$
|f(z)| \leq \frac{M}{R^{m}}|z|^{m} \quad\left(z \in \mathbb{U}_{R}\right) .
$$

The equality can hold only if $f(z)=e^{i \theta}\left(M / R^{m}\right) z^{m}$, where $\theta$ is constant.

Theorem $\mathbf{A}$ (see [5]). If the function $f$ is regular in the unit disk $\mathbb{U}, f(z)=z+a_{2} z^{2}+\cdots$ and

$$
\left(1-|z|^{2}\right)\left|\frac{z f^{\prime \prime}(z)}{f^{\prime}(z)}\right| \leq 1,
$$

for all $z \in \mathbb{U}$, then the function $f$ is univalent in $\mathbb{U}$.

Theorem B (see [6]). If $f \in \mathscr{A}$ satisfies the condition

$$
\left|\frac{z^{2} f^{\prime}(z)}{(f(z))^{2}}-1\right| \leq 1 \quad(z \in \mathbb{U}),
$$

then $f$ is univalent in $\mathbb{U}$.

\section{Main Results}

Theorem 3. Let $f_{i}, g_{i} \in \mathscr{A}$, where $g_{i}$ satisfies the condition

$$
\left|\frac{z^{2} g_{i}^{\prime}(z)}{\left(g_{i}(z)\right)^{2}}-1\right| \leq 1
$$

$\alpha_{i} \in \mathbb{C}$, and $M_{i}>0$ for all $i=1, \ldots, n$. If

$$
\begin{gathered}
\left|\frac{f_{i}^{\prime \prime}(z)}{f_{i}^{\prime}(z)}\right| \leq 1, \\
\left|g_{i}(z)\right|<M_{i}, \\
\left|\alpha_{i}\right| \leq \frac{9}{2 n \sqrt{3}\left(1+2 M_{i}^{2}\right)},
\end{gathered}
$$

for all $i=1, \ldots, n$, then the integral operator $I_{n, \alpha}(f, g)$ defined by (10) is in the univalent function class $\mathcal{S}$.
Proof. From (10), we obtain

$$
I_{n, \alpha}^{\prime}(f, g)(z)=\left(f_{1}^{\prime}(z) e^{g_{1}(z)}\right)^{\alpha_{1}} \cdots\left(f_{n}^{\prime}(z) e^{g_{n}(z)}\right)^{\alpha_{n}}
$$

for $z \in \mathbb{U}$. This equality implies that

$$
\begin{aligned}
\ln I_{n, \alpha}^{\prime}(f, g)(z)= & \alpha_{1}\left(\ln f_{1}^{\prime}(z)+g_{1}(z)\right) \\
& +\cdots+\alpha_{n}\left(\ln f_{n}^{\prime}(z)+g_{n}(z)\right) .
\end{aligned}
$$

By differentiating the above equality, we get

$$
\begin{aligned}
\frac{I_{n, \alpha}^{\prime \prime}(f, g)(z)}{I_{n, \alpha}^{\prime}(f, g)(z)}= & \alpha_{1}\left(\frac{f_{1}^{\prime \prime}(z)}{f_{1}^{\prime}(z)}+g_{1}^{\prime}(z)\right) \\
& +\cdots+\alpha_{n}\left(\frac{f_{n}^{\prime \prime}(z)}{f_{n}^{\prime}(z)}+g_{n}^{\prime}(z)\right),
\end{aligned}
$$

or equivalently

$$
\begin{aligned}
\frac{z I_{n, \alpha}^{\prime \prime}(f, g)(z)}{I_{n, \alpha}^{\prime}(f, g)(z)}= & \alpha_{1}\left(\frac{z f_{1}^{\prime \prime}(z)}{f_{1}^{\prime}(z)}+z g_{1}^{\prime}(z)\right) \\
& +\cdots+\alpha_{n}\left(\frac{z f_{n}^{\prime \prime}(z)}{f_{n}^{\prime}(z)}+z g_{n}^{\prime}(z)\right) .
\end{aligned}
$$

So we find

$$
\begin{aligned}
& \left(1-|z|^{2}\right)\left|\frac{z I_{n, \alpha}^{\prime \prime}(f, g)(z)}{I_{n, \alpha}^{\prime}(f, g)(z)}\right| \\
& \quad=\left(1-|z|^{2}\right)\left|\sum_{i=1}^{n} \alpha_{i}\left(\frac{z f_{i}^{\prime \prime}(z)}{f_{i}^{\prime}(z)}+z g_{i}^{\prime}(z)\right)\right| \\
& \quad \leq\left(1-|z|^{2}\right) \sum_{i=1}^{n}\left|\alpha_{i}\right|\left(|z|\left|\frac{f_{i}^{\prime \prime}(z)}{f_{i}^{\prime}(z)}\right|+\left|z g_{i}^{\prime}(z)\right|\right) \\
& \quad \leq\left(1-|z|^{2}\right) \sum_{i=1}^{n}\left|\alpha_{i}\right|\left(|z|+\left|z g_{i}^{\prime}(z)\right|\right) \\
& \quad \leq\left(1-|z|^{2}\right) \sum_{i=1}^{n}\left|\alpha_{i}\right|\left(|z|+\left|\frac{z^{2} g_{i}^{\prime}(z)}{g_{i}^{2}(z)}\right| \frac{\left|g_{i}^{2}(z)\right|}{|z|}\right) .
\end{aligned}
$$

From the hypothesis, we have $\left|g_{i}(z)\right|<M_{i}(z \in \mathbb{U} ; i \in$ $\{1, \ldots, n\})$; then by the general Schwarz lemma, we obtain that

$$
\left|g_{i}(z)\right| \leq M_{i}|z| \quad(z \in \mathbb{U} ; i \in\{1, \ldots, n\}) .
$$


Thus we have

$$
\begin{aligned}
& \left(1-|z|^{2}\right)\left|\frac{z I_{n, \alpha}^{\prime \prime}(f, g)(z)}{I_{n, \alpha}^{\prime}(f, g)(z)}\right| \\
& \quad \leq\left(1-|z|^{2}\right) \sum_{i=1}^{n}\left|\alpha_{i}\right|\left(|z|+\left|\frac{z^{2} g_{i}^{\prime}(z)}{g_{i}^{2}(z)}\right| M_{i}^{2}|z|\right) \\
& \quad \leq\left(1-|z|^{2}\right) \sum_{i=1}^{n}\left|\alpha_{i}\right||z|\left(1+\left|\frac{z^{2} g_{i}^{\prime}(z)}{g_{i}^{2}(z)}-1\right| M_{i}^{2}+M_{i}^{2}\right) \\
& \quad \leq|z|\left(1-|z|^{2}\right) \sum_{i=1}^{n}\left|\alpha_{i}\right|\left(1+2 M_{i}^{2}\right) .
\end{aligned}
$$

Let us define the function $F:[0,1] \rightarrow \mathbb{R}, F(x)=x\left(1-x^{2}\right)$, $x=|z|$. Then we obtain

$$
F(x) \leq \frac{2 \sqrt{3}}{9},
$$

for all $x \in[0,1]$. It follows from (26) that

$$
|z|\left(1-|z|^{2}\right) \leq \frac{2 \sqrt{3}}{9} .
$$

Hence from (18), (25), and (27) we find

$$
\left(1-|z|^{2}\right)\left|\frac{z I_{n, \alpha}^{\prime \prime}(f, g)(z)}{I_{n, \alpha}^{\prime}(f, g)(z)}\right| \leq 1 \text {. }
$$

By applying Theorem A for the function $I_{n, \alpha}(f, g)$, we prove that $I_{n, \alpha}(f, g)$ is in the univalent function class $\mathcal{S}$.

Setting $n=1, f_{1}=f, g_{1}=g$, and $\alpha_{1}=\alpha$ in Theorem 3, we obtain the following consequence of Theorem 3 .

Corollary 4 (see [3]). Let $f, g \in \mathscr{A}$, where $g$ satisfies the condition

$$
\left|\frac{z^{2} g^{\prime}(z)}{(g(z))^{2}}-1\right| \leq 1
$$

$\alpha \in \mathbb{C}$, and $M>0$. If

$$
\begin{gathered}
\left|\frac{f^{\prime \prime}(z)}{f^{\prime}(z)}\right| \leq 1, \\
|g(z)|<M, \\
|\alpha| \leq \frac{9}{2 \sqrt{3}\left(1+2 M^{2}\right)},
\end{gathered}
$$

then the integral operator $I_{1}(f, g)$ defined by (11) is in the univalent function class $\mathcal{S}$.

Theorem 5. Let $f_{i}, g_{i} \in \mathscr{A}$, where $g_{i} \in \mathscr{B}\left(\mu_{i}, \beta_{i}\right)\left(\mu_{i} \geq 0,0 \leq\right.$ $\left.\beta_{i}<1\right), \alpha_{i} \in \mathbb{C}$, and $M_{i} \geq 1$ for all $i=1, \ldots, n$. If

$$
\begin{aligned}
& \left|\frac{f_{i}^{\prime \prime}(z)}{f_{i}^{\prime}(z)}\right| \leq 1, \\
& \left|g_{i}(z)\right|<M_{i},
\end{aligned}
$$

$$
0<\sum_{i=1}^{n}\left|\alpha_{i}\right|\left(1+\left(2-\beta_{i}\right) M_{i}^{\mu_{i}}\right) \leq 1,
$$

then the integral operator $I_{n, \alpha}(f, g)$ defined by $(10)$ is in the class $\mathscr{K}(\delta)$, where

$$
\delta=1-\sum_{i=1}^{n}\left|\alpha_{i}\right|\left(1+\left(2-\beta_{i}\right) M_{i}^{\mu_{i}}\right) .
$$

Proof. From (22), we have

$$
\begin{aligned}
\frac{z I_{n, \alpha}^{\prime \prime}(f, g)(z)}{I_{n, \alpha}^{\prime}(f, g)(z)}= & \alpha_{1}\left(\frac{z f_{1}^{\prime \prime}(z)}{f_{1}^{\prime}(z)}+z g_{1}^{\prime}(z)\right) \\
& +\cdots+\alpha_{n}\left(\frac{z f_{n}^{\prime \prime}(z)}{f_{n}^{\prime}(z)}+z g_{n}^{\prime}(z)\right) .
\end{aligned}
$$

It follows that

$$
\begin{aligned}
& \left|\frac{z I_{n, \alpha}^{\prime \prime}(f, g)(z)}{I_{n, \alpha}^{\prime}(f, g)(z)}\right| \\
& \quad \leq \sum_{i=1}^{n}\left|\alpha_{i}\right||z|\left(\left|\frac{f_{i}^{\prime \prime}(z)}{f_{i}^{\prime}(z)}\right|+\left|g_{i}^{\prime}(z)\right|\right) \\
& \quad \leq \sum_{i=1}^{n}\left|\alpha_{i}\right||z|\left(1+\left.\left|g_{i}^{\prime}(z)\left(\frac{z}{g_{i}(z)}\right)^{\mu_{i}}\right| \frac{g_{i}(z)}{z}\right|^{\mu_{i}} \mid\right) .
\end{aligned}
$$

Since $g_{i} \in \mathscr{B}\left(\mu_{i}, \beta_{i}\right),\left|g_{i}(z)\right|<M_{i}$ for all $i=1, \ldots, n$, applying general Schwarz lemma and using (35), we obtain

$$
\begin{aligned}
& \left|\frac{z I_{n, \alpha}^{\prime \prime}(f, g)(z)}{I_{n, \alpha}^{\prime}(f, g)(z)}\right| \\
& \quad \leq \sum_{i=1}^{n}\left|\alpha_{i}\right||z|\left(1+\left|g_{i}^{\prime}(z)\left(\frac{z}{g_{i}(z)}\right)^{\mu_{i}} M_{i}^{\mu_{i}}\right|\right) \\
& \quad \leq \sum_{i=1}^{n}\left|\alpha_{i}\right||z|\left(1+\left|g_{i}^{\prime}(z)\left(\frac{z}{g_{i}(z)}\right)^{\mu_{i}}-1\right| M_{i}^{\mu_{i}}+M_{i}^{\mu_{i}}\right) \\
& \quad<\sum_{i=1}^{n}\left|\alpha_{i}\right|\left(1+\left(2-\beta_{i}\right) M_{i}^{\mu_{i}}\right)=1-\delta .
\end{aligned}
$$

This implies that the integral operator $I_{n, \alpha}(f, g) \in \mathscr{K}(\delta)$.

Setting $\mu_{1}=\mu_{2}=\cdots=\mu_{n}=1$ in Theorem 5, we have the following.

Corollary 6. Let $f_{i}, g_{i} \in \mathscr{A}$, where $g_{i} \in \mathcal{S}^{*}\left(\beta_{i}\right)\left(0 \leq \beta_{i}<1\right)$, $\alpha_{i} \in \mathbb{C}$, and $M_{i} \geq 1$ for all $i=1, \ldots, n$. If

$$
\begin{aligned}
& \left|\frac{f_{i}^{\prime \prime}(z)}{f_{i}^{\prime}(z)}\right| \leq 1, \\
& \left|g_{i}(z)\right|<M_{i},
\end{aligned}
$$

for all $i=1, \ldots, n$, and if 


$$
0<\sum_{i=1}^{n}\left|\alpha_{i}\right|\left(1+\left(2-\beta_{i}\right) M_{i}\right) \leq 1,
$$

then the integral operator $I_{n, \alpha}(f, g)$ defined by $(10)$ is in the class $\mathscr{K}(\gamma)$, where

$$
\gamma=1-\sum_{i=1}^{n}\left|\alpha_{i}\right|\left(1+\left(2-\beta_{i}\right) M_{i}\right) .
$$

Setting $\mu_{1}=\mu_{2}=\cdots=\mu_{n}=0$ in Theorem 5, we have the following.

Corollary 7. Let $f_{i}, g_{i} \in \mathscr{A}$, where $g_{i} \in \mathscr{R}\left(\beta_{i}\right)\left(0 \leq \beta_{i}<1\right)$, $\alpha_{i} \in \mathbb{C}$, and $M_{i} \geq 1$ for all $i=1, \ldots, n$. If

$$
\begin{aligned}
& \left|\frac{f_{i}^{\prime \prime}(z)}{f_{i}^{\prime}(z)}\right| \leq 1, \\
& \left|g_{i}(z)\right|<M_{i}
\end{aligned}
$$

for all $i=1, \ldots, n$, and if

$$
0<\sum_{i=1}^{n}\left|\alpha_{i}\right|\left(3-\beta_{i}\right) \leq 1,
$$

then the integral operator $I_{n, \alpha}(f, g)$ defined by $(10)$ is in the class $\mathscr{K}(\lambda)$, where

$$
\lambda=1-\sum_{i=1}^{n}\left|\alpha_{i}\right|\left(3-\beta_{i}\right) .
$$

Setting $n=1, f_{1}=f, g_{1}=g$, and $\alpha_{1}=\alpha$ in Theorem 5, we obtain the following consequence of Theorem 5 .

Corollary 8 (see [3]). Let $f, g \in \mathscr{A}$, where $g \in \mathscr{B}(\mu, \beta)(\mu \geq$ $0,0 \leq \beta<1), \alpha \in \mathbb{C}$, and $M \geq 1$. If

$$
\begin{gathered}
\left|\frac{f^{\prime \prime}(z)}{f^{\prime}(z)}\right| \leq 1, \\
|g(z)|<M, \\
0<|\alpha|\left(1+(2-\beta) M^{\mu}\right) \leq 1,
\end{gathered}
$$

then the integral operator $I_{1}(f, g)$ defined by (11) is in the class $\mathscr{K}(\rho)$, where

$$
\rho=1-|\alpha|\left(1+(2-\beta) M^{\mu}\right) .
$$

\section{Conflict of Interests}

The authors declare that there is no conflict of interests regarding the publication of this paper.

\section{References}

[1] B. A. Frasin and J. M. Jahangiri, "A new and comprehensive class of analytic functions," Analele Universităţii din Oradea, vol. 15, pp. 59-62, 2008.
[2] B. A. Frasin and M. Darus, "On certain analytic univalent functions," International Journal of Mathematics and Mathematical Sciences, vol. 25, no. 5, pp. 305-310, 2001.

[3] N. Ularu and D. Breaz, "Univalence criterion and convexity for an integral operator," Applied Mathematics Letters, vol. 25, no. 3, pp. 658-661, 2012.

[4] Z. Nehari, Conformal Mapping, Dover, New York, NY, USA, 1975.

[5] J. Becker, "Löwnersche differentialgleichung und quasikonform fortsetzbare schlichte funktionen," Journal für die Reine und Angewandte Mathematik, vol. 255, pp. 23-43, 1972.

[6] S. Ozaki and M. Nunokawa, "The Schwarzian derivative and univalent functions," Proceedings of the American Mathematical Society, vol. 33, pp. 392-394, 1972. 


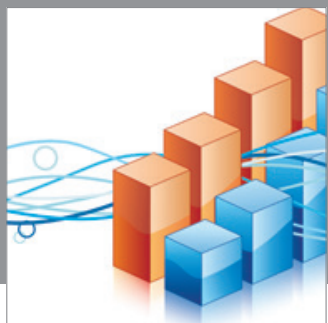

Advances in

Operations Research

mansans

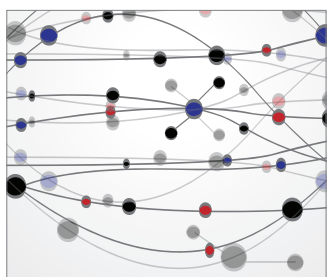

The Scientific World Journal
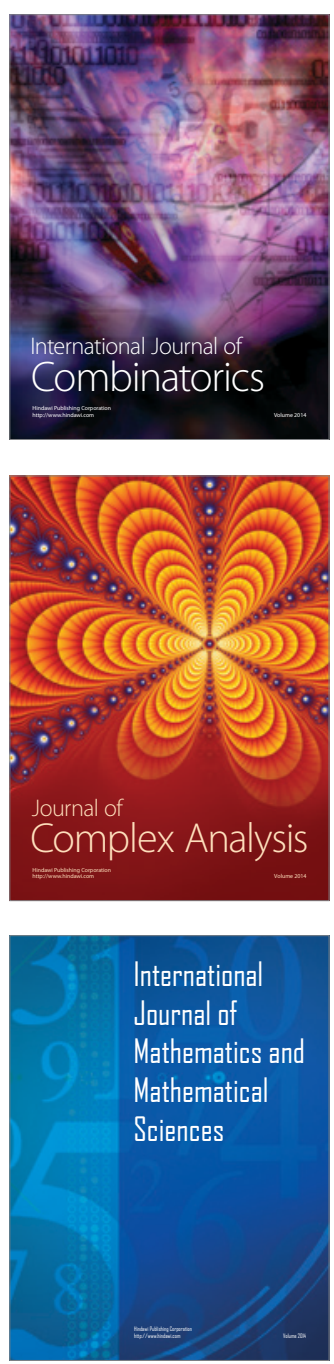
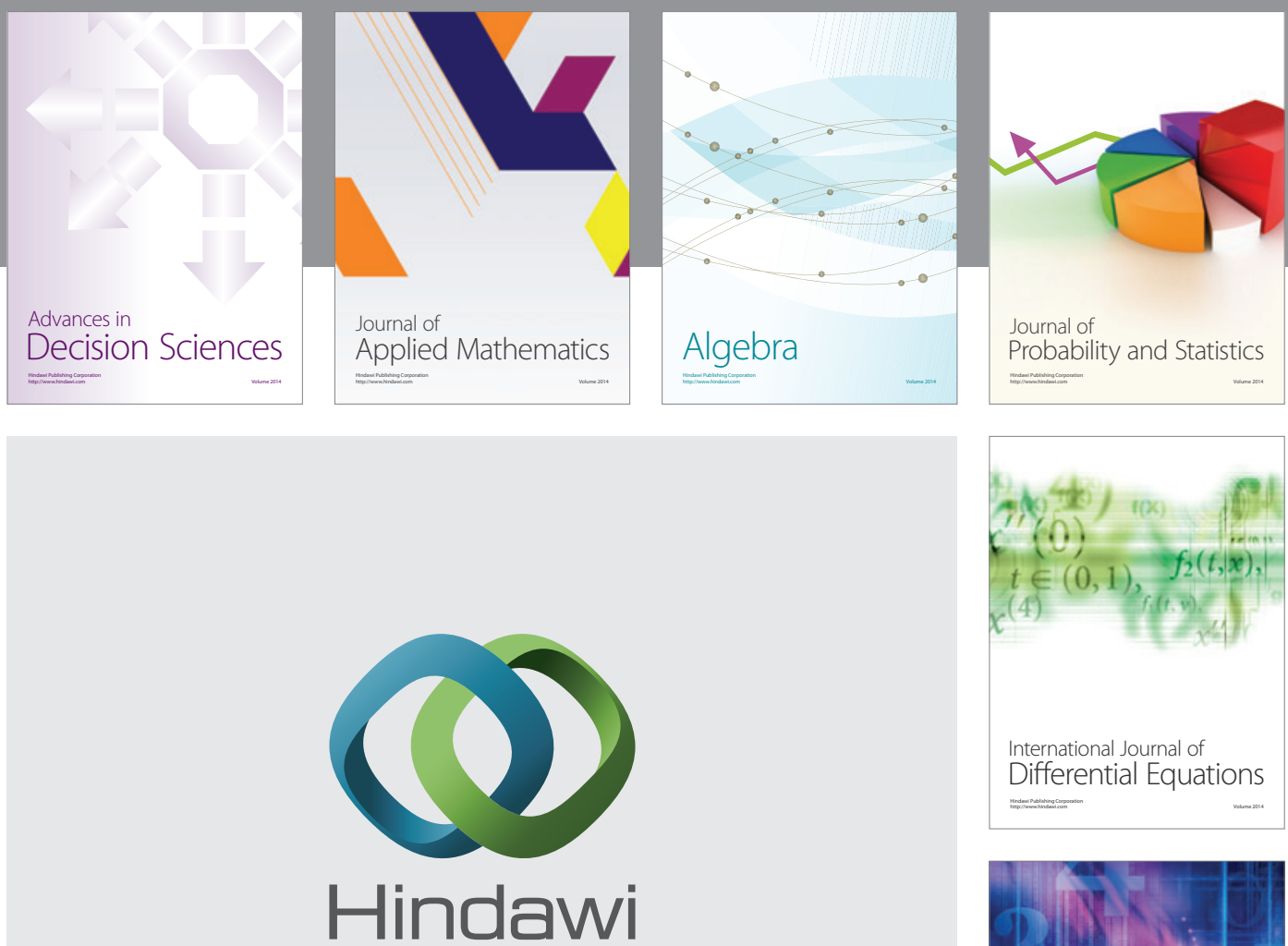

Submit your manuscripts at http://www.hindawi.com
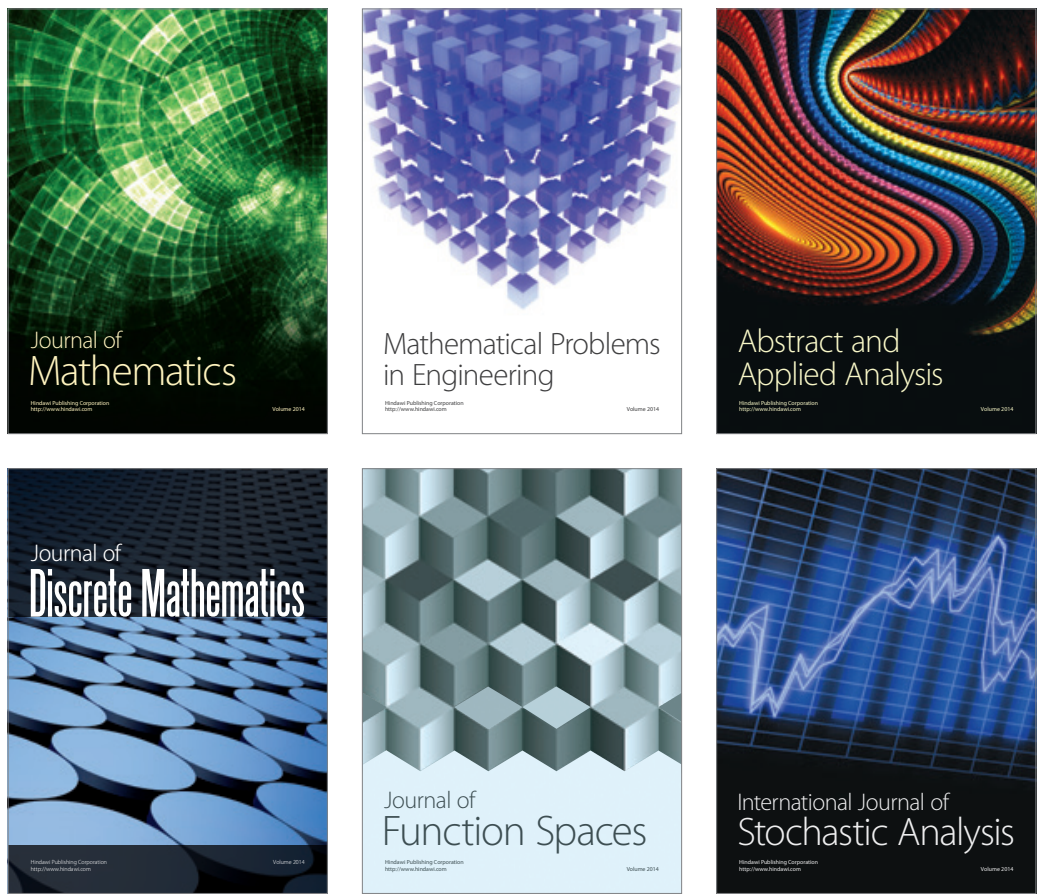

Journal of

Function Spaces

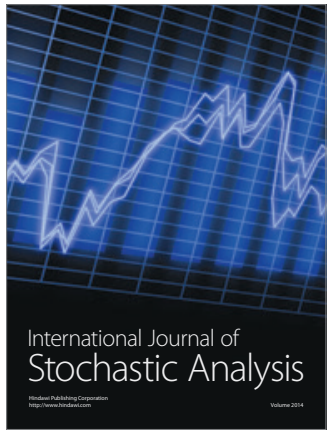

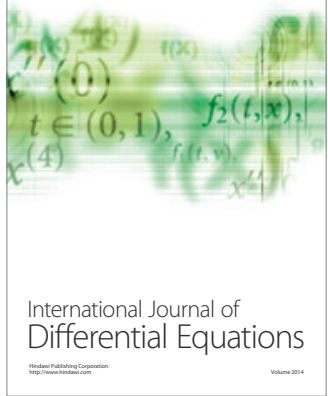
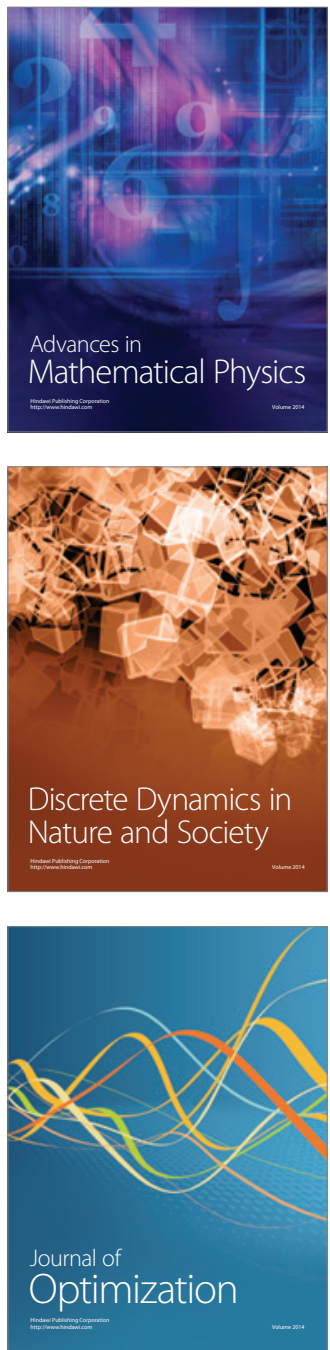